\title{
Algorithms for mesh repairing to represent automobile parts
}

\author{
Weiguo $\mathrm{LI}^{1,2^{*}}$, Wenhua $\mathrm{YE}^{1}$, Wenliang $\mathrm{CHEN}^{1}$ \\ 1. College of Mechanical and Electrical Engineering, Nanjing University of Aeronautics and Astronautics, Nanjing 210016, China \\ 2. School of Mathematics and Information Technology, Nanjing Xiaozhuang University, Nanjing 210000, China
}

\begin{abstract}
In representing automobile parts with mesh in the field of reverse engineering or finite element generation, the mesh reconstruction and data exchanging between different CAD/CAM systems often introduce many invisible topological and geometrical errors into mesh. These artifacts can cause serious problems in subsequent operations such as finite element analysis, reverse engineering, animation, and simulation. In this study we propose a practical method for repairing topological and geometrical errors on mesh. First, coincident vertices during mesh input are removed, followed by the identification of non-manifold vertices and edges. The non-manifold vertices are modified, and the facets having non-manifold edges are removed. Finally, faces that have the wrong orientations in the mesh are re-oriented. Experiments show that our methods can eliminate most common mesh errors quickly and effectively. The refined mesh can be properly used in subsequent operations.
\end{abstract}

Key words: mesh; topological repairing; geometrical repairing; non-manifold vertices; non-manifold edges; automobile parts; finite element

(C) 2011 JMT. All rights reserved.

\section{Introduction}

$\mathrm{M}$ esh, a popular way of representing 3D objects such as automobile parts, is extensively used in $\mathrm{CAD} / \mathrm{CAE}$ system. However, the exchange of mesh data between different $\mathrm{CAD} / \mathrm{CAE}$ software systems (such as Dynaform, LS-PrePost, CATIA, Pro/E, SolidWorks, 3DS, ImageWare, and GeoMagic) and different file formats (such as STL, WRL, and OBJ) can introduce many errors. Operations such as deletion, merging, simplification or deformation of mesh would also generate errors. These errors may cause major problems for the implementation of ray-tracing, finite element analysis, subdivision surface generation, and other high-level CAE or surfacing operations.

Errors on mesh are classified into two typical types: topological errors and geometric errors. Topological error is judged by its manifoldness; and geometric error is often retrieved by the statistical information from mesh data, such as coincident vertices, wrongly oriented or overlapped facets.

To ensure the manifoldness, Gueziec [1] proposed a cutting and stitching method for mesh repairing. The method does not take the local geometrical information

Received Jun. 27, 2011; revision accepted Sep. 27, 2011

*Corresponding author. E-mail: wli@resurf3d.com (W.G. LI)

(C) 2011 JMT. All rights reserved doi: 10.3969/j.issn.2095-087X.2011.04.006 into account, and sometimes the mesh gets distorted severely after repairing. Forest [2] proposed an algorithm to keep volumetric tetrahedral manifoldness instead of triangulated manifoldness after removing a tetrahedron from mesh. Akleman [3] introduced topological modeling operators INSERTEDGE and DELETEEDGE based on topological graph theory [4]. Using these operators, they designed an interactive system [5] for modeling 2manifold mesh. Based on planar graph theory, Wood et al. [6], Guskov et al. [7], and Leong et al. [8-9] presented algorithms to de-noising the topology. Zhou et al. [10] presented a method for repairing topological errors on solid models by using skeletons. Ju's method [11] introduced a target shape by drawing 3D line skeleton and modified the topology of the 3D source model by the skeleton.

To remove coincident vertices, Cui [12] proposed a method based on multi-table schema. Coincidence is detected only when different vertices point to the same index in the table. Desbrun [13] used diffusion and curvature flow to de-noise irregular meshes. They confidently rely on weighted mean curvature which normally is not accurate in noisy mesh. Fleishman [14] proposed a simple image-based mesh de-noising method. However, it is only applicable to manifold meshes, and it may introduce self-intersection.

In this paper, we will propose a method for repairing errors in mesh. In order to extend our algorithm to a widespread application, we will discuss the topic based on general mesh rather than automobile part only. 


\section{System overview}

Our mesh repairing method originates itself from the motivation to provide a repaired mesh for generating subdivision surface, which is used extensively in animation and reverse engineering. Most subdivision algorithms in the literature such as Catmull-Clark and DooSabin schema require that local region of the mesh be able to map a simple disk. It means that the topology of the mesh must be manifold. Since the quality of the subdivision surface relies strongly on the raw mesh, the quality of the raw mesh is extremely important.

The algorithm for repairing mesh essentially consists of two preprocessing phases: topological error correction and geometrical error fixing. Fig. 1 shows the overview of the error repairing method.

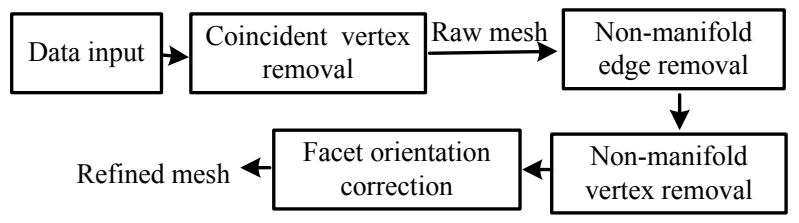

Fig. 1 Flow chart of our mesh repairing method

(1) Data input: Initially, mesh is read into the computer from a file. The file format can be OBJ, STL, WRL, etc. Here mesh is represented using indexed Face\&Edge Set structure; i.e., the input triangular facet structure stores the indices of the three vertices and three edges. All faces are stored in a face array. All vertices are stored in a vertex array.

(2) Coincident vertex removal: If different vertices have the same three coordinates (in certain precision range), they are treated as coincident vertices. They should be removed immediately after file reading, and then a raw mesh is obtained. Vertex coincidence is a geometric error that often results in data translations.

(3) Non-manifold edge removal: If an edge is adjacent to more than two neighboring facets, it is called a non-manifold edge (or singular edge). In fact, most data structures for representing mesh cannot process nonmanifold edge well. The best way is to delete all related facets which introduce this edge and fill the holes caused by this deletion through a hole-filling operation.

(4) Non-manifold vertex removal: If all facets connecting to a vertex are not connected each other by their adjacent edges, the vertex is called a non-manifold vertex (or singular vertex). Here no face should be deleted. Instead, the gap between these facets will be filled.

(5) Facet orientation correction: Orientation defines the normal direction to the face. To get the normal of correct facets for rendering or other operations, wrongly orientated facets should be corrected.

\section{Topological error repairing}

Manifoldness is an important requirement for many mesh operations. For finite element analysis, nonmanifold mesh often leads to ill-conditioned linear system of equations. For subdivision surface generation, non manifold skeleton mesh often leads to selfintersection surfaces.

In topological term, mesh is a straight-line graph embedded in $\mathbf{R}^{3}$. We call the facets in mesh that share the umbrella of the vertex. The number of facets in the umbrella is called the valence of the vertex. If the umbrella is a chain (open region) or a cycle (closed region), the vertex is regular. If the umbrella (1) has multiple chains, (2) has multiple cycles, or (3) is grouped by chains and cycles, the vertex is a singular (non-manifold) vertex. Fig. 2 shows some regular and singular vertices.

If an edge is adjacent to exactly one facet, it's called a boundary edge. If the number of adjacent facets is two, it is called a regular edge. Otherwise, it is called a singular (non-manifold) edge. Samples of boundary, regular and singular edges are shown in Fig. 3.

A topological mesh is manifold if each vertex is locally homeomorphous to the plane, and each vertex on the boundary is locally homeomorphous to the halfplane. Here, we give the definition of manifoldness for mesh using singular property.

Definition 1 A mesh is manifold if and only if it has no singular edge and no singular vertex. Otherwise, it is a non-manifold mesh.

Fig. 4 shows two non-manifold meshes.

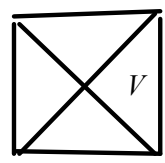

(a)

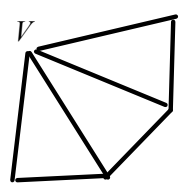

(b)

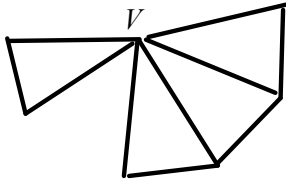

(c)

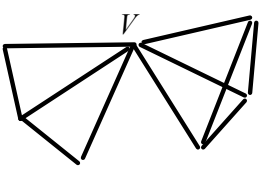

(d)

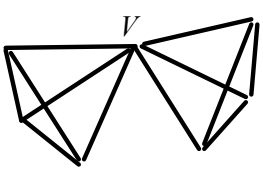

(e)

Fig. 2 Umbrella of a vertex: (a) a regular vertex with one cycle region (closed); (b) a regular vertex with one chain region; (c) a singular vertex with two chain regions; (d) a singular vertex with one chain region and one cycle region; and (e) a singular vertex with two cycle regions 


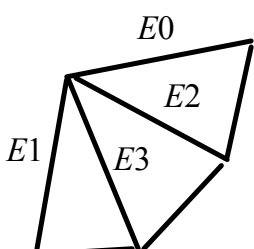

(a)

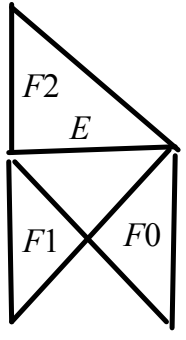

(b)
Fig. 3 Edge classifications: (a) $E 0$ and $E 1$ are boundary edges, while $E 2$ and $E 3$ are regular edges; (b) $E$ is a singular edge: $F 0, F 1$, and $F 2$ share $E$ as a common edge.

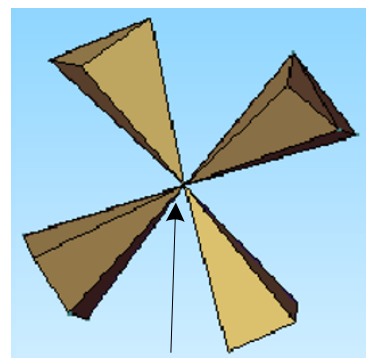

Nonmanifold vertex

(a)

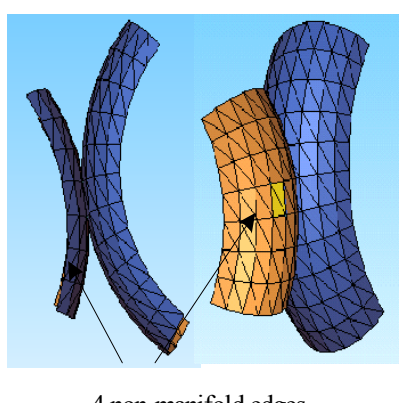

(b)
Fig. 4 Real-world examples of non-manifold mesh: (a) a nonmanifold mesh with one singular vertex; (b) a non-manifold mesh caused by four singular edges (highlighted in yellow)

\subsection{Detection and modification of non-manifold edges}

This section discusses the detection and removing of singular edges. As mentioned in Section 2, we use "Indexed Face\&Edge Set" data structure to represent the mesh. The edge structure stores two neighboring facets' ID (left facet ID and right facet ID). If an edge is singular, the number of adjacent facets is more than two. Fig. 5 outlines an algorithm and flow chart for detecting singular edges.

Facet and edge both have a singularity flag. Algorithm 1 (Fig. 5) is based on the fact that an edge is a regular or boundary vertex only when the adjacent facet and edge will point to each other directly through indices stored in their data structures. In Table 1, if a singular edge is detected, a flag will be set in the edge data structure, and the defected facet will also be labeled. For example, in Fig. 6, three faces $F 0, F 1$ and $F 2$ share an edge $E$. But edge $E$ only stores $F 0$ and $F 1$ as its adjacent faces. Therefore, $E$ will be treated as a singular edge, and $F 2$ is labeled as a defected facet.

The simplest method to eliminate the singular edges is to delete all defected faces which cause the singularity. Using this method, in Fig. 6, F2 will be deleted and edge $E$ will then become regular.

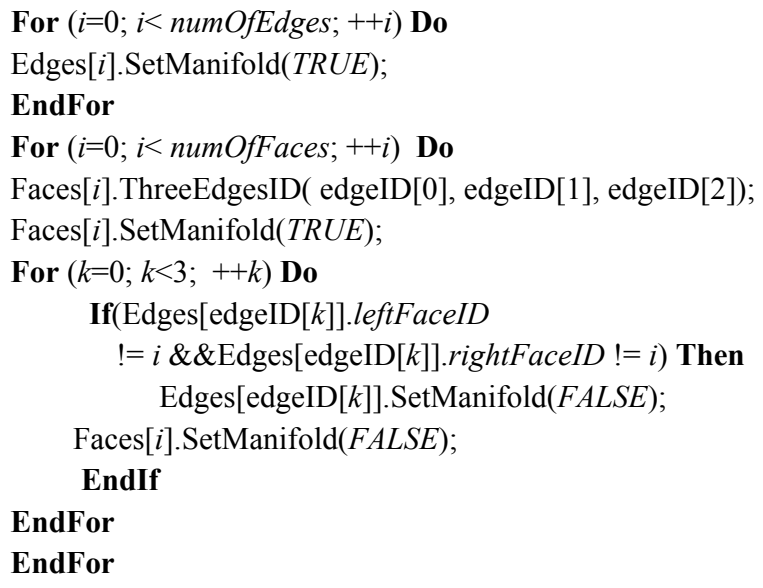

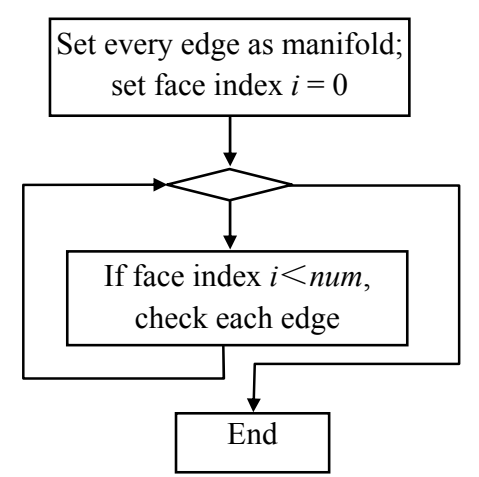

(b)

Fig. 5 Algorithm 1 (a) and the flow chart (b) for detecting singular edges

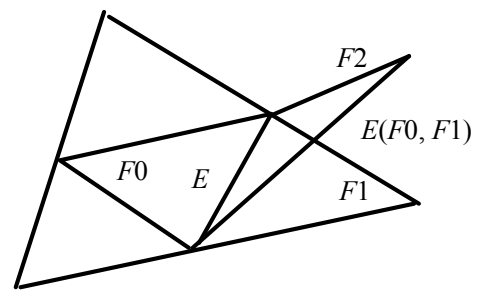

Fig. 6 A singular edge $E$

Although defected facets indeed cause topological errors, they may be parts of a well-formed shape. Simply deleting defected facets can lead to serious deformations on the mesh. Fig. 7 shows such an example. Facets $F 0$ and $F 1$ are overlapped (they have the same vertices and edges) in Fig. 7(a). The overlapping causes edges E0, $E 1$, and $E 2$ to become singular. If the adjacent facets, stored in the data structures of $E 0, E 1$ and $E 2$, are $F 0$ and $F 1$, we will end up deleting facets $F 2, F 3$, and $F 4$ 


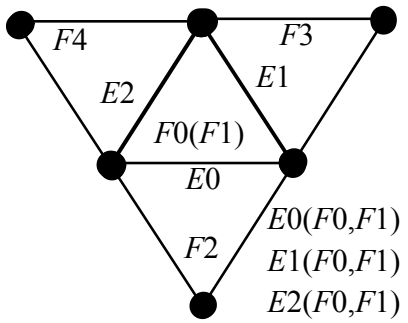

(a)

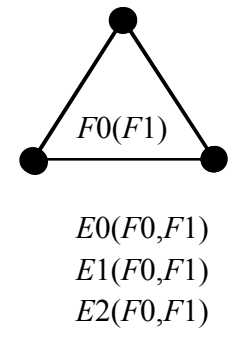

(b)

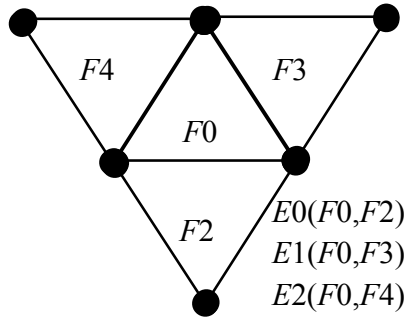

(c)

Fig. 7 Choosing facets with singular edges to delete: (a) a raw local region, it has three singular edges; (b) improper deleting strategy; and (c) better deleting strategy.

using the above simple deleting method, and get the result in Fig. 7(b), which is not a desirable one.

Example shows that the overlapped facets that are resulted by tessellation and data exporting from 3D systems produce a large portion of singular edges in realworld applications. To avoid the result in Fig. 7(b), and prior to delete any facet, we should identify whether two adjacent facets stored in the data structure of the singular edge, are overlapped or not. If they are overlapped, one of the overlapped facets will be deleted, and the adjacent face index stored in the singular edge, will be modified accordingly. Fig. 7(c) shows the result of this improved deletion strategy.

In real-world applications, singular edges often are crimped in a rather small region, and multiple facet deletions often introduce some small holes. We use the stitch strategy proposed in Gueziec [1] to fill these small holes.

\section{Procedure IsVertexManifold(VerIndex $i d x$ ) \\ Begin}

Vertices $[i d x]$.GetAdjacentFacesID(verAdjFaceIDs);

boundTris.push(verAdjFaceIDs.pop());

While (!boundTris.empty()) Do

triId $x=$ boundTris.pop( $)$;

size $=$ Faces $[$ triId $x]$.GetAdjacentFacesID(neibTris);

For $(k=0 ; k<$ size; $++k)$ Do

If $\left(\mathrm{m} \_\mathrm{pFaces}[\mathrm{neibTris}[\mathrm{k}]] . \mathrm{IsVertexOnFace}(\mathrm{idx})\right)$

Then

$$
\text { If }((\text { index }=\text { FindInArray }(\text { verAdjFaceIDs, }
$$
neibTris[k]))!=NULL) Then

RemoveElement(verAdjFaceIDs, index); boundTris.push(neibTris[k]);

\section{EndIf \\ EndIf}

EndFor

EndWhile

return verAdjFaceIDs.IsEmpty();

(a)

\subsection{Detection and modification of non-manifold vertices}

Many mesh operations, such as hole filling and mesh smoothing, are based on finding all adjacent vertices to a given vertex. Singular vertex (refer to Fig. 2) presents a major challenge for these mesh operation algorithms. We cannot fix the singular vertices by simply deleting the facets. For example, in Fig. 4(a), the labeled vertex is non-manifold, but deleting any facet will destroy the mesh seriously. We present an algorithm for fixing singular vertices in Fig. 8 and Fig. 9. In our algorithm, cycle regions of a singular vertex will not be modified. They are treated as rational point in geometry. Only the singular vertices whose umbrellas contain multiple chains will be modified. The vertices in Fig. 2(c), (d), and (e) will be modified, but the vertices in Fig. 2(a) and (b) will be kept unchanged. In this study, we focus on fixing the singular vertices similar to Fig. 2(c), and propose an algorithm to merge all chain regions into one.

Fig. 8 Algorithm 2 (a) and flow chart (b) for detecting singular vertices 
The algorithm for detecting singular vertices is shown in Fig. 8.

The following algorithm is for merging chains in the umbrella lists:

Step 1: Cluster the umbrella of singular vertex into independent chain regions.

Step 2: Find two boundary edges in each chain region.

Step 3: Calculate angles between boundary edges which are located in different chain regions, and put them into an angle list.

Step 4: Sort angles in the ascending order.

Step 5: Choose the two boundary edges with the minimal angle as the edges for the chain merging. Bridge a triangular facet between them, and merge the two regions with the two selected boundary edges. Remove all angles related to the two edges from the angle list.

Step 6: If the number of left chain regions is less than two, terminate the algorithm; otherwise, repeat Step 5 and Step 6.

Fig. 9 shows an example of chain merging. Initially, singular vertex $V$ has three chains. They are labeled as Region 0 , Region 1 and Region 2 . The boundary edges in the chain regions are labeled as E00, E01 for Region 0, E10, E11 for Region 1 and E20, E21 for Region 2 . The angles between two boundary edges are listed in the right side in Fig. 9(a), and are sorted in the ascending order. The angle between edge E11 and E20 is the minimal one. We bridge a triangular facet $N F 0$ between edge E11 and E20, and merge Region 1 and Region 2 into one region. All angle items related to $E 11$ and $E 20$ will be removed from the angle list. Repeating the above process and continuing the merging operation, we get Fig. 9(b) and Fig. 9(c). A regular vertex $V$ is the final result, which is shown in Fig. 9(c).

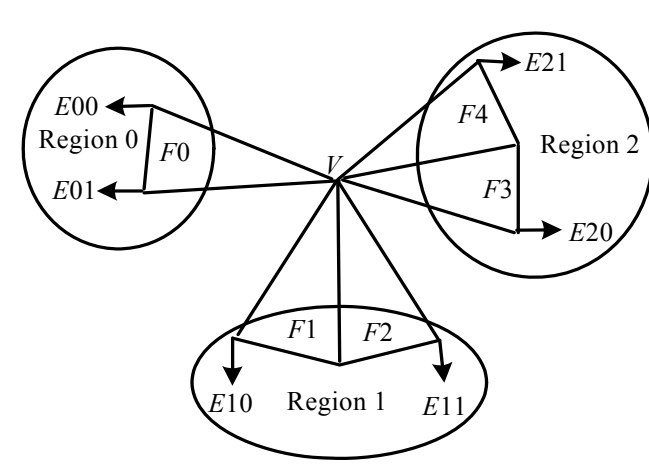

(a)

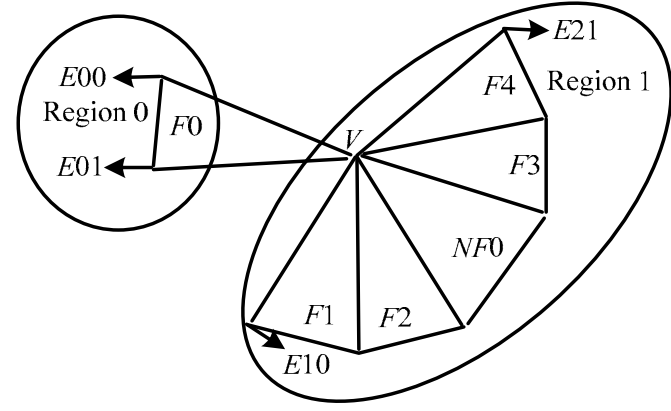

(b)
Angles between boundary edges in diffrent regions (in ascending order): $A(E 00, V, E 10)$

$A(E 00, V, E 11)$

$A(E 00, V, E 20)$

$A(E 00, V, E 21)$

$A(E 01, V, E 10)$

$A(E 01, V, E 11)$

$A(E 01, V, E 20)$

$A(E 01, V, E 21)$

$A(E 10, V, E 20)$

$A(E 10, V, E 21)$

$A(E 11, V, E 20)$ (minimal)

$A(E 11, V, E 21)$

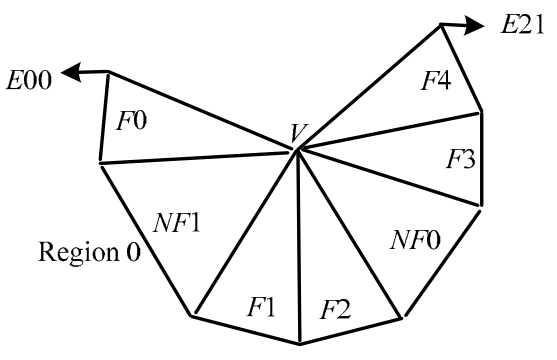

(c)

Fig. 9 Examples of fixing non-manifold vertex using chain merging algorithm: (a) singular vertex with three chains; (b) adding $N F 0$ after merging Regions 1 and 2; (c) regular vertex obtained by adding $N F 0$ and $N F 1$

\section{Geometrical repairing}

Well-formed shape is an important requirement for many applications such as animation and finite element analysis. Coincidence of vertices and wrong orientation of facets are two major geometrical errors in mesh. This section focuses on detecting and correcting the coincident vertices and wrong facet orientations.

\subsection{Coincident vertex detection and removal}

Two vertices with the same coordinates (within certain precision range) in $3 \mathrm{D}$ space are treated as being coincident. Coincident vertices will introduce corrupt triangles with zero edge length, and lead to failures in geometric calculations of the facet.

It is obvious that detection of coincident vertices can 
be converted to a searching problem. Compared to linear searching and binary searching, hash table searching is the quickest one. To use hash table search, we should define the length of hash table $L$, the hash function $H$, and the key of the hash function $K$.

Based on the fact that most vertices will never be coincident with more than 2 vertices in mesh, the length of the hash table is simply defined as $L=N / 2$, where $N$ is the number of vertices in the mesh. If the coordinates of the vertex in space is represented by three float variables (representing $X, Y$, and $Z$ coordinates), since each float has four bytes in memory, we can treat them as an unsigned integer data, and the key of hash function is defined as $K=x+y+z$. Hash function is simply defined as $H(K)=K \bmod L$. Table 1 shows the results of deletion of the coincident vertices using hash table on a P5 2.0 GHZ, 1.0 G RAM machine.

Obtained coincident vertices will be labeled. The vertices can be simply removed, and all adjacent edges and faces can be deleted. Instead of using the above simple deletion strategy, we present a dynamic adjusting method for fixing coincident vertices without removing any existing facets (Fig. 10). In Fig. 10(a), vertices $V 2$ and $V 3$ are coincident. They introduce two corrupt triangles $F 1$ and $F 3$. First, we translate vertex $V 3$ to the middle of the edge $(V 1, V 2)$. The changed region is shown in Fig. $10(\mathrm{~b})$, and it can be seen that triangle $F 3$ becomes regular. We swap edge $(V 1, V 2)$ and edge $(V 0, V 3)$ between triangles $F 0$ and $F 1$, and triangle $F 1$ also becomes regular. The final result is shown in Fig. 10(c).

Table 1 The results of coincident vertices removal using hash table

\begin{tabular}{cccc}
\hline$N$ & $L$ & Coincident vertices & Time (s) \\
\hline 6353 & 3176 & 33 & 0.164 \\
49961 & 24980 & 253 & 0.277 \\
122318 & 61159 & 611 & 0.369 \\
356127 & 178063 & 1780 & 0.433 \\
412921 & 206460 & 2064 & 0.733 \\
\hline
\end{tabular}

\subsection{Correction of wrongly-oriented facets}

A mesh is oriented only if each facet is oriented such that the two vertices of each shared edge in two faces are listed in opposite orders. A mesh is called orientable if orientations of its facets can be chosen such that each edge is oriented in two opposite directions. A mesh is re-oriented correctly after eliminating all errors of nonmanifold edges.

In Fig. 11(a), two facets, labeled as $F 0$ and $F 1$, are wrongly-oriented because the orders of $V 1$ and $V 2$ in $F 0$ and $F 1$ are the same. In Fig. 11(b), $F 0$ and $F 1$ are oriented correctly. Wrongly oriented face generates opposite normal directions, and causes problems in rendering and other operations. In Fig. 11(a), the normal of $F 0$ points outward while the normal of $F 1$ points inward. In Fig. 11(b), all normal vectors point outward. Fig. 11(c) shows the result of rendering the wrongly oriented mesh, while Fig. 11(d) is the correct result. The algorithm for correct re-orientation of a mesh is shown in Fig. 12.

\section{Experiments}

In this study, we present the repairing methods for defected meshes based on detecting and fixing of nonmanifold/singular edges and vertices, and of geometric errors such as vertex coincidence and wrong-orientation of facets. The refined meshes can be properly used in post-processing operations, such as finite element analysis, and reverse engineering surface generation.

All of algorithms presented in this study are implemented in $\mathrm{C}++$ and have been incorporated into an existing 3D system named LS-PrePost, which is a program for preparing finite element data for LS-DYNA developed by LSTC. One of the main uses of the algorithm is to ensure that STL files are always imported as correct manifolds and to reduce geometric errors. Our algorithm has been successfully tested on many commercially available models. Fig. 13 shows an example of auto- mobile part for our proposed algorithm. Fig. 14 shows another example for mesh topology repaired by the presented algorithm.

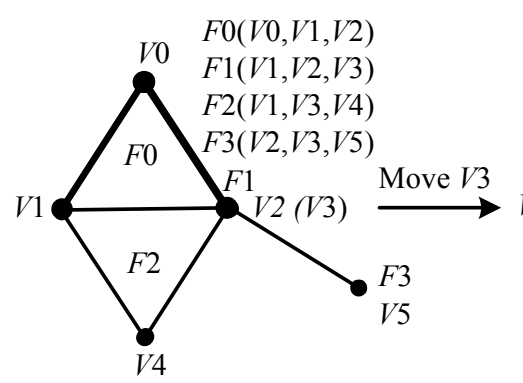

(a)

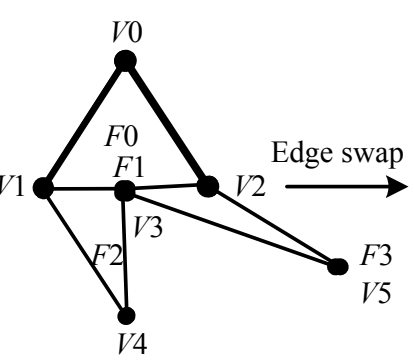

(b)

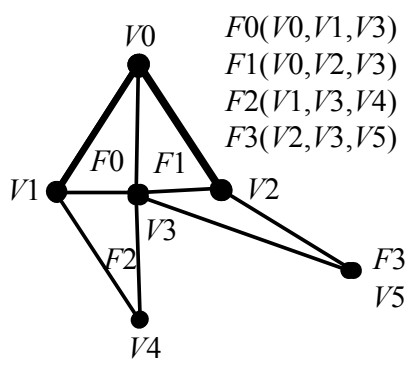

(c)

Fig. 10 Dynamical modification of coincident vertices: (a) 4 facets with 2 coincident vertices; (b) new vertex $V 3$ locating at the middle of the edge $(V 1, V 2)$; (c) 4 facets without coincident vertex 


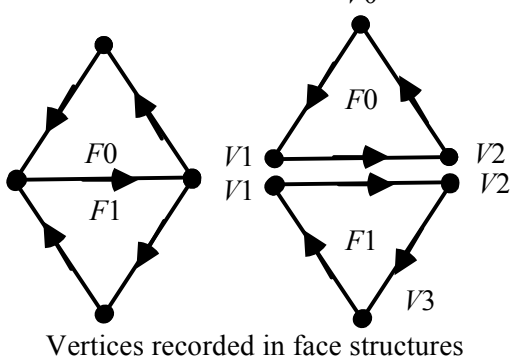

$F 0(V 0, V 1, V 2)$

$F 1(V 3, V 1, V 2)$

(a)

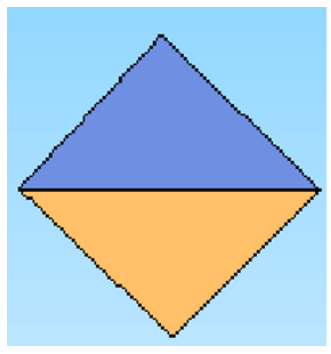

(c)
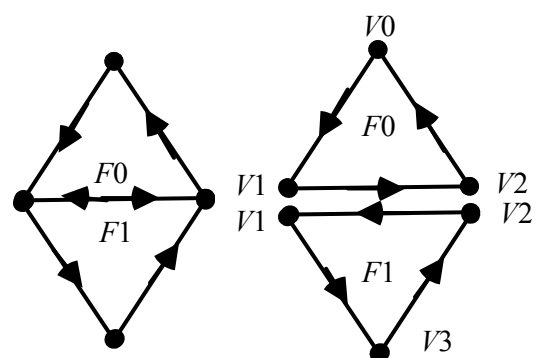

Vertices recorded in face structures

$$
\begin{aligned}
& F 0(V 0, V 1, V 2) \\
& F 1(V 3, V 2, V 1)
\end{aligned}
$$

(b)

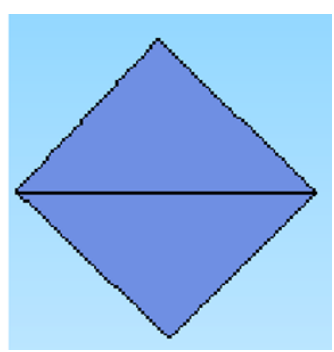

(d)

Fig. 11 Correction of wrongly-oriented facets: (a) wrongly orientated facets; (b) correctly oriented facets; (c) rendering of (a); (d) rendering result of $(b)$

\section{Procedure Reorientation}

Begin

While (TRUE) Do

For $(i=0 ; i<$ NumOfFaces; $++i)$ Do

If (bUsed $[i])$ Then continue EndIf

list.insert_back $(i)$

bUsed $[i]=T R U E$

break

\section{EndFor}

If $(i==$ NumOfFaces $)$ Then return EndIf

While (list.IsEmpty ()$==F A L S E)$ Do

faceId = list.remove_front ();

Faces[faceId]. ThreeVerticesID(v[0], v[1], v[2]);

For $(i=0 ; i<3 ;++i)$ Do

edgeId $=$ GetEdgeID $(\mathrm{v}[i], \mathrm{v}[(i+1) \% 3])$;

faceIdNext $=$ Edges[edgeId].GetAnotherFaceId(faceId);

If $($ faceIdNext $==-1 \|$ bUsed[faceIdNext])

Then continue EndIf

If (Faces[faceIdNext].IsWrongOriented (v[( $i+1) \% 3]$,

$\mathrm{v}[i]))$ Then

Faces[faceIdNext].ReverseOrientation();

\section{EndIf}

bUsed [faceIdNext $]=$ TRUE;

faceIdList.insert_back(faceIdNext);

\section{EndFor}

\section{EndWhile}

End

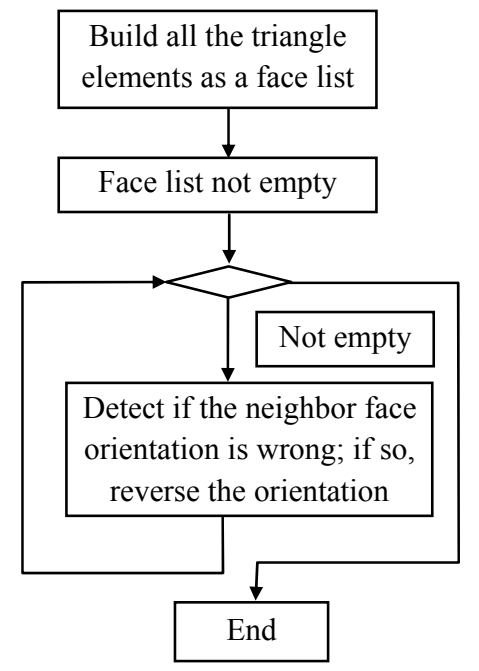

(a)

(b)

Fig.12 Algorithm 3 (a) and flow chart (b) for re-orienting a mesh 


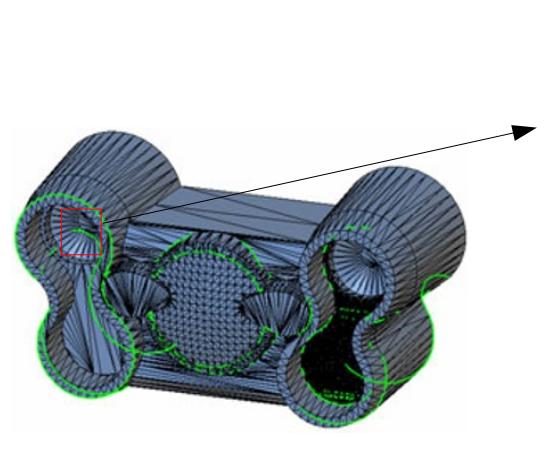

(a)

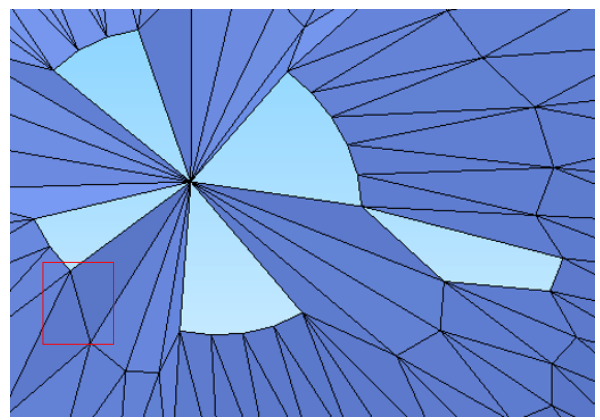

(c)

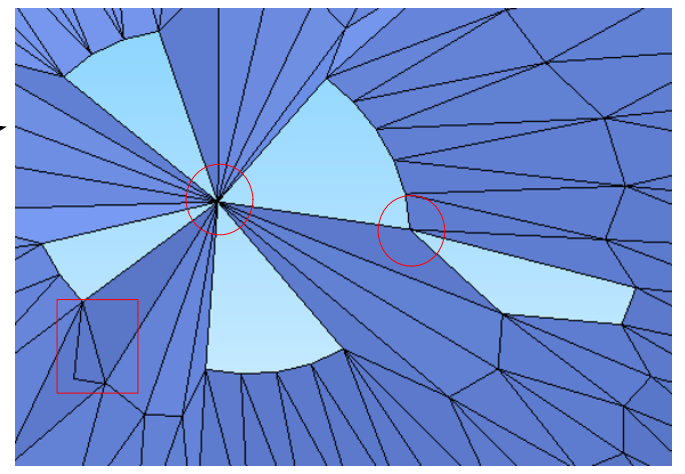

(b)

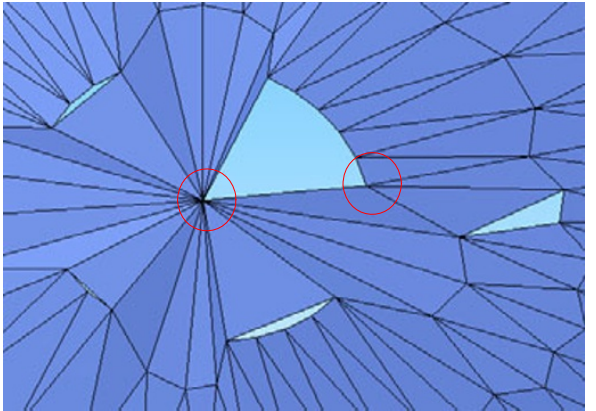

(d)

Fig. 13 The automobile part model: (a) The highlighted poly lines represent the errors of singular vertices and edges, and coincident vertices. (b) The local view. Two singular vertices were detected and labeled as red circle. One singular edge was detected and labeled as red rectangle. (c) After removing a redundant triangle, the edge is regular. (d) After merging chains of singular vertices, two vertices became regular.

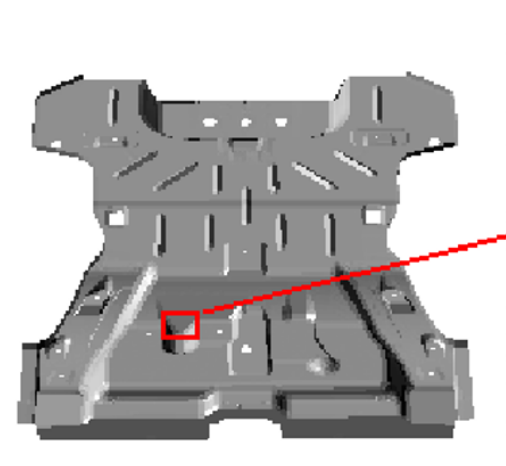

(a)

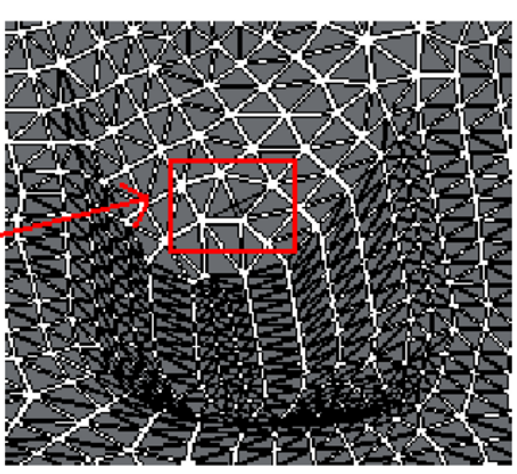

(b)

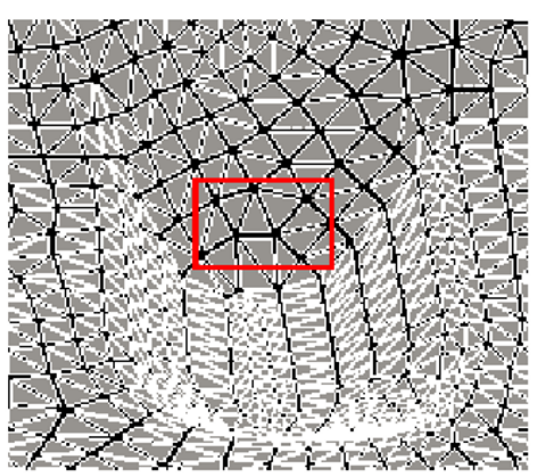

(c)

Fig. 14 The automobile floor part finite element mesh model: (a) The highlighted poly lines represent the errors of topology. This error was produced in the process of finite element mesh generation from NURBS surfaces. (b) The local view shows that there is a triangle element overlapping the two triangle elements. This triangle element has two non-manifold edges and results in the error of topology. (c) After the triangle element was detected and removed, the topology became correct.

\section{Conclusion}

The proposed method automatically and intelligently converts a non-manifold mesh to a manifold one. Furthermore, it quickly detects and corrects the wronglyoriented faces, and identifies and removes coincident vertices. Experiments show that the method can elimi- nate most errors in defected meshes. The refined meshes can be properly used in post-processing operations, such as finite element analysis and reverse engineering. We have successfully unfolded 3D finite element mesh into 2D mesh and converted 3D digital laser scanned mesh into NURBS surface by repairing connectivity errors a prior based on the algorithms presented in this paper 
[15]. In the future research, we will adopt the algorithms to clean up 3D arbitrary mesh models for mesh parameterization, e.g. Periodic Global Parameterization.

\section{References}

[1] A. Gueziec, G. Taubin, F. Lazarus, et al., Cutting and stitching: converting sets of polygons to manifold surfaces, IEEE Transactions on Visualization and Computer Graphics, 2011, 7(2): 136-151.

[2] C. Forest, H. Delingette, N. Ayache, Removing tetrahedra from a manifold mesh, In: Proceedings of IEEE Computer Animation, Geneva, Switzerland, Jun. 19-21, 2002: 225-232.

[3] E. Akleman, J. Chen, Guaranteeing the 2-manifold property for meshes with doubly linked face list, International Journal of Shape Modeling, 1999, 5(2): 149-177.

[4] J. Chen, Algorithmic graph embeddings, Theoretical Computer Science, 1997, 181(2): 247-266.

[5] E. Akleman, J. Chen, V. Srinivasan, An interactive system for modeling 2-manifold meshes, In: Proceedings of International Conference on Shape Modeling and Applications, Banff, Alberta, May 17-22, 2002: 43-50.

[6] Z.J. Wood, H. Hoppe, M. Desbrun, et al., Removing excess topology from isosurfaces, ACM Transactions on Graphics, 2004, 23(2): 190-208.

[7] I. Guskov, Z.J. Wood, Topological noise removal, In: Proceedings of Graphics Interface, Toronto, Canada, 2001: 19-26.

[8] K.F. Leong, C.K. Chua, Y.M. Ng, A study of stereo- lithography file errors and repair. Part 1 . Generic solution, International Journal of Advanced Manufacturing Technology, 1996, 12(6): 407-414.

[9] K.F. Leong, C.K. Chua, Y.M. Ng, A study of stereolithography file errors and repair. Part2. Special cases, International Journal of Advanced Manufacturing Technology, 1996, 12(6): 415-422.

[10] Q.Y. Zhou, T. Ju, S.M. Hu, Topology repair of solid models using skeletons, IEEE Transactions on Visualization and Computer Graphics, 2007, 13(4): 675-685.

[11] T. Ju, Q.Y. Zhou, S.M. Hu, Editing the topology of 3d models by sketching, ACM Transactions on Graphics (TOG)_Proceedings of ACM SIGGRAPH 2007, 2007, 26(3): 42.1-42.9.

[12] S.B. Cui, Y.S. Zhang, S.Y. Liang, et al., The algorithm and application of sorting for fast filtering redundancy vertex in STL solid, Chinese Mechanical Engineering, 2001, 12(2): 173-174 (in Chinese).

[13] M. Desbrun, M. Meyer, P. Schroder, et al., Implicit fairing of irregular meshes using diffusion and curvature flow, In: Proceedings of the 26th Annual Conference on Computer Graphics and Interactive Techniques, Los Angeles, Aug. 8-13, 1999: 317-324.

[14] S. Fleishman, I. Drori, D. Cohen-Or, Bilateral mesh denoising, ACM Transactions on Graphics (TOG)Proceedings of ACM SIGGRAPH 2003, 2003, 22(3): 950-953.

[15] X.H. Zhu, D. Bhalsod, L. Zhang, et al., An enhanced one-step metal forming solution in LS-DYNA, http:// www.dynalook.com/fea-newsletters/fea-newsletter-2011/ fea-newsletter-august-2011.pdf, 2011-08-30.

(Editor: Dongju CHEN) 